Particle Accelerators, 1990, Vol. 29, pp. 145-152 Reprints available directly from the publisher Photocopying permitted by license only
(C) 1990 Gordon and Breach, Science Publishers, Inc. Printed in the United States of America

\title{
THE VACUUM SYSTEM OF THE HERA ELECTRON STORAGE RING
}

\author{
R. BALLION, J. BOSTER, W. GIESSKE, H. HARTWIG, D. JAGNOW, \\ R. KOSE, J. KOUPTSIDIS, G. SCHUMANN and M. SCHWARTZ \\ Deutsches Elektronen-Synchrotron DESY, D-2000 Hamburg, Germany
}

Abstract The $6.3 \mathrm{~km}$ long vacuum system of the HERA electron ring is fabricated by brazing tubes made from the alloy CuSn2. This copper alloy absorbs synchrotron light more efficiently than aluminium and therefore reduces radiation shielding problems. The system is mainly pumped by integrated sputter-ion pumps using the fields of the dipole and quadrupole magnets. They provide a maximum linear pumping speed of $301 / \mathrm{s}$ per meter. The impedance of the beam environment is kept small by minimizing the dimensions of the pump slots and avoiding steps of greater than $1 \mathrm{~mm}$ inside the vacuum chamber. A vacuum pressure in the $10^{-9}$ mbar range was achieved some days after assembly of the vacuum system and without bake out in situ. A beam life time of more than one hour was observed during the first test runs of HERA.

\section{INTRODUCTION}

Since 1961 much experience has been gained on vacuum performance and the requirements of electron storage rings up to beam energies of 25 GeV. ${ }^{1}$ Problems due to intense synchrotron light, such as compcnent overheating or high gas desorption rates turned out not to be serious. Overheating can be avoided by fabricating the vacuum components from nigh thermal conductivity materials and by using water cooling. Also, high gas desorption rates are significantly reduced after an adequate operating time, due to the cleaning effect of synchrotron light. ${ }^{1}$ This also makes superfluous the bake-out in situ, which is normally used to reduce gas desorption.

It seems that attention has to be paid to two other effects. These are the possibility of the escape of synchotron light through the vacuum chamber walls ${ }^{2}$ and the excitation of transverse single bunch instabilities ${ }^{3}$, due to interaction of the beam with the environment. ${ }^{4}$ Both effects are more serious for the new high energy storage rings HERA ${ }^{5}$ and LEP ${ }^{6}$, due to the shift of the synchrotron radiation spectrum towards higher energy ${ }^{1}$ and to the higher bunch currents which are necessary to fill the increased circumference. 
The vacuum chambers of nearly all electron storage rings are made from aluminium alloys which can be inexpensively extruded to form vacuum tight tubes consisting of more than one channel which accommodate the beam, pumps, and water cooling. In addition, aluminium provides high thermal conductivity, low degassing rate, and good weldability. The relatively high transparency to the $x$ - and $y$-rays did not impede its use in existing storage rings, since the escaping radiation power was too low to cause thermal effects on the surrounding magnets. Their iron yokes have therefore been used as an additional inexpensive radiation shield.

For the new large storage rings HERA ${ }^{5}$ and LEP ${ }^{6}$, there is, however, a significant increase in the synchrotron light power escaping through an aluminium chamber. To avoid unacceptable heating of the magnets, either less transparent materials have to be used instead of aluminium, or a lead shield some millimeters thick has to be soldered onto the aluminum vacuum chamber. ${ }^{6}$ In the last case, the magnet gaps must be enlarged. This increases the production and operation costs of the magnets.

Therefore, a copper alloy was preferred for the fabrication of the HERA vacuum chambers, because of its relativly high absorption coefficient and because of the high thermal conductivity. The $4 \mathrm{~mm}$ thick copper chamber reduces the escaping synchrotron radiation power in HERA, at the nominal energy of $30 \mathrm{GeV}$, to less than $8 \%$, compered to about $50 \%$ for aluminum. ${ }^{5}$ At these low power levels, it is still possible to use the magnet yokes as an additional radiation shield. Thus additional lead shields and magnet gap enlargement can be avoided. As in the case of small rings, only the magnet openings have to be provided with lead shields(Fig. 1).

In addition to design principles this report presents an outline of the main vacuum components, including fabrication materials and techniques. Finally, the first operational experience with the HERA vacuum system will be described.

\section{THE HERA VACUUM SYSTEM}

The arcs of the HERA electron ring, having a total length of $4.8 \mathrm{~km}$, 
consist of 400 modules. Each module consists of a $9 \mathrm{~m}$ long bending magnet, a quadrupole magnet, a sextupole magnet and a steering magnet. A single copper vacuum chamber spans the total module length of about $12 \mathrm{~m}$. Fig. 1 shows the vacuum chamber inside a bending and quadrupole magnet respectively. The chamber is also provided with small, $5 \mathrm{~mm}$ thick, lead radiation shields to close just the magnet joke gaps. The modules were prepared outside of the HERA tunnel, including the assembly of vacuum chambers with radiation shields etc.. Such completed modules were then taken into the tunnel and installed in their predetermined positions.

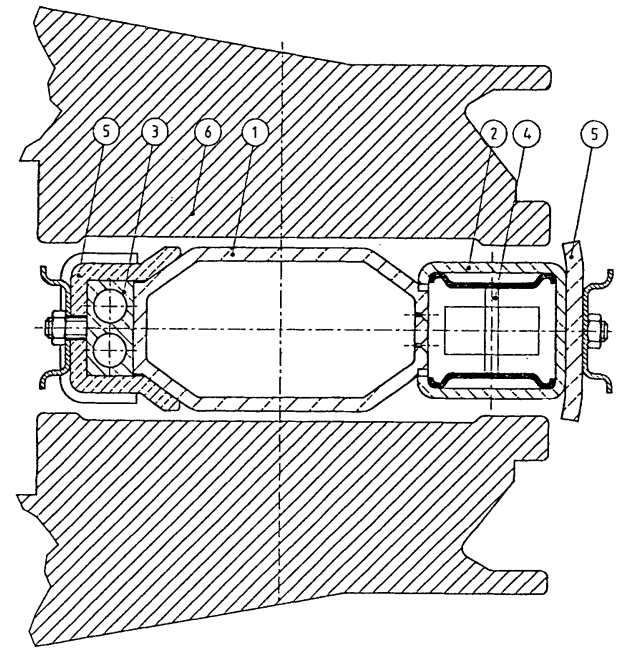

a) Bending Magnet

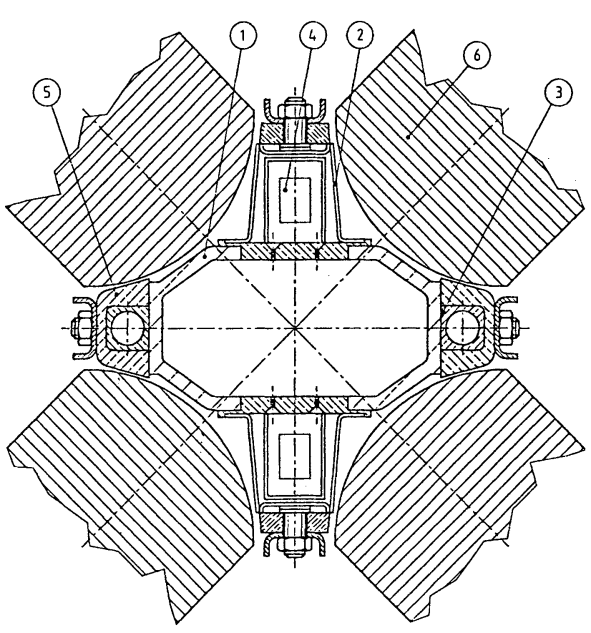

b) Quadrupole Magnet

FIGURE 1 : Vacuum chamber cross sections inside the magnets. $1=$ beam pipe; 2 = pump channel/appendix; 3 = cooling water; 4 = integrated pump; $5=$ lead shields; 6 = iron magnet yokes

The vacuum system is mainly pumped by integrated sputter-ion pumps which use the magnetic field of the dipoles and quadrupoles. The main beam pipe has a cross section of $80 \times 40 \mathrm{~mm}^{2}$. Over the $9 \mathrm{~m}$ length of the bending magnet it is provided with a longitudinal brazed channel for the integrated dipole pump(Fig. 1a). Similar pumps have been also installed inside the quadrupole magnets by brazing on stainless steel appendices, both on the top and bottom of the beam pipe(Fig. 1b). The quadrupole pumps are mainly foreseen for pumping the $3 \mathrm{~m}$ length of the vacuum pipe between two dipoles, which has too low a conductance to be otherwise effectively pumped. Both integrated pump types are designed ${ }^{7}$ 
to give a maximum effective linear pumping speed of about 30 $1 . \mathrm{s}^{-1} \cdot \mathrm{m}^{-1}$. This is sufficient to make the vacuum performance of HERA similar to that of PETRA. Additionally, every second module is provided with a standard $801 . \mathrm{s}^{-1}$ sputter-ion pump to maintain the vacuum when the magnets are switched off.

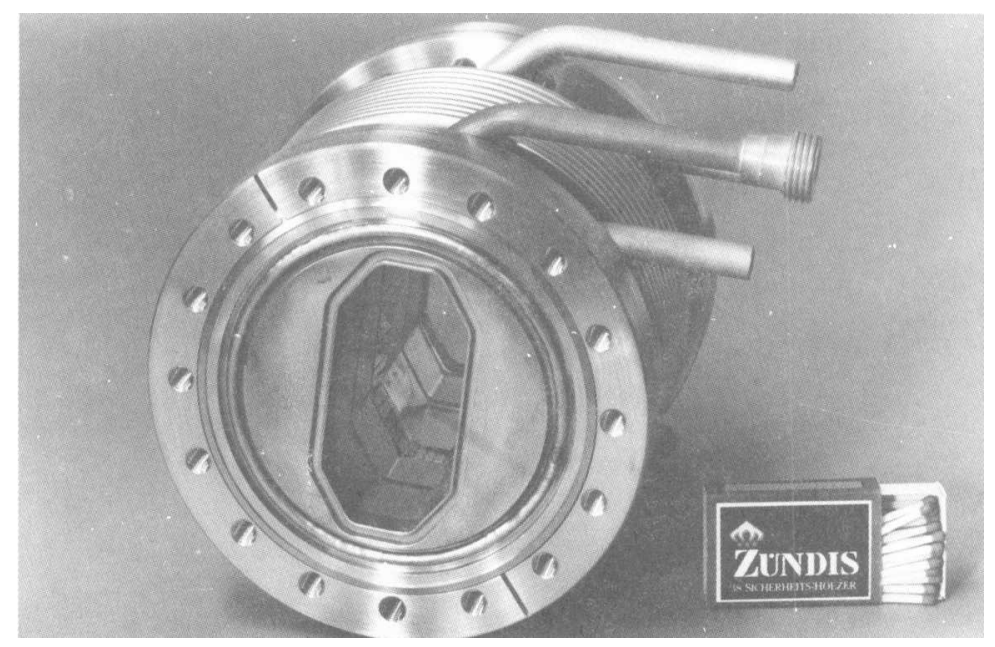

FIGURE 2 : The HERA expansion joint between two chambers An rf-shield with sliding contacts screens the joint bellow.

The vacuum chambers of two modules are connected together by a $140 \mathrm{~mm}$ long expansion joint(fig. 2) having a bellow for the mechanical tolerances, and the thermal expansion. The bellow is short circuited internally by using an rf-shield with sliding contacts. The remaining steps inside the beam environment are kept to less than $1 \mathrm{~mm}$ in order to reduce the transverse beam impedance. ${ }^{3}$ The expansion joint must also be intensively water cooled, since it is illuminated by the synchrotron light.

The vacuum chambers of the remaining $1.5 \mathrm{~km}$ long straight sections are also mainly made from copper. Their lengths, lying between .5 and $12 \mathrm{~m}$, are chosen to accommodate the straight section components such as rf-cavities, spin rotators etc.. The vacuum chambers close to the experiments are especially designed ${ }^{7}$ in order to reduce the backgrounds in the experiments, caused by the synchrotron radiation photons. They are either provided locally with special collimators to shield the experiment from the main part of incident photons, or they are asymmetrically enlarged to allow the remaining 
photons to strike the chamber walls far away from the experiment. The straight sections are pumped with the same pumping speed as the arcs, by using either integrated pumps installed in the quadrupole magnets, or additional standard $801 . \mathrm{s}^{-1}$ sputter-ion pumps.

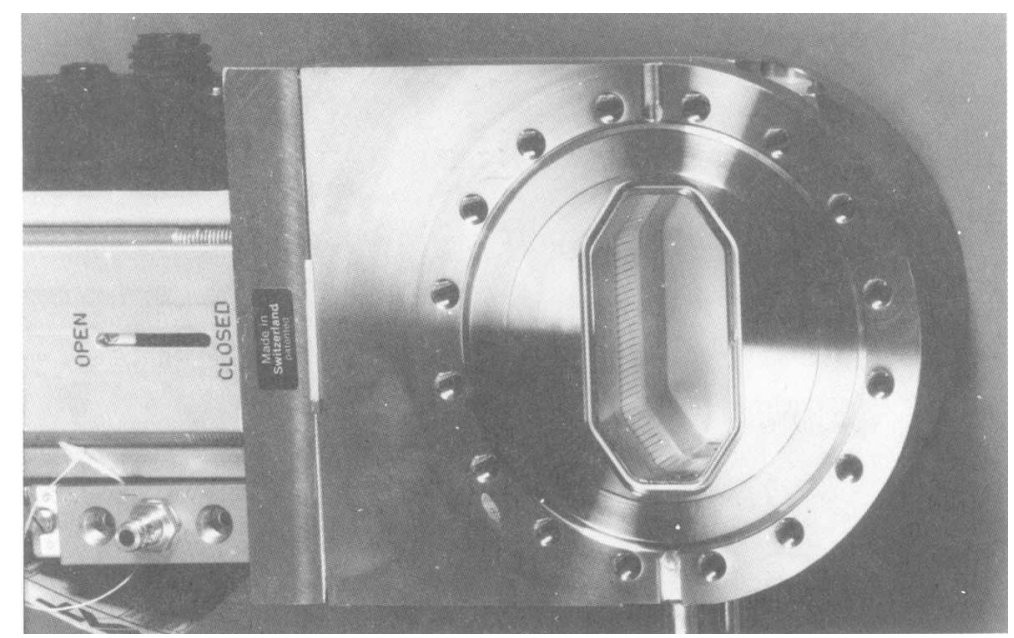

FIGURE 3 : The new HERA compact gate valve in open position.

The HERA electron vacuum system is divided by gate valves into 42 vacuum sections having various lengths up to $200 \mathrm{~m}$. The patented gate valve has been especially developed for HERA. It is the most compact valve with low beam transverse impedance to be developed so far Fig. 3 shows such a valve in the open position. The same valve can be seen installed between two vacuum sections in HERA(Fig. 4). Each vacuum section is provided with a turbomolecular pump for pump-down before the sputter-ion pumps are started. Groups of four such pumps are driven by a small, newly developed, inexpensive high voltage power supply. The current of these power supplies serves to monitor the pressure distribution in HERA.

\section{MATERIALS AND FABRICATION TECHNIQUES}

The use of copper alloys for the vacuum chamber fabrication implied the development of new techniques, since it is not possible las for aluminium) to extrude vacuum tight copper tubes consisting of more 
than one channel. The HERA vacuum chambers are therefore fabricated by brazing together the various single tubes needed to accommodate the beam, the integrated pumps, and the water cooling. The use of single tubes also allows optimal machining of the pump slots which connect the beam channel with the pumps, and whose use is preferable for reducing the $r f$ parasitic mode losses and the transversal beam pipe impedance seen by the beam. ${ }^{4}$

Only the water cooling tubes of the vacuum chambers are made from ordinary oxygen free copper. On the contrary, the main beam tube and the pump channel are solid-drawn from the copper bronze CuSn2. The bronze contains $2 \%$ of tin to add stiffness to the copper and therefore

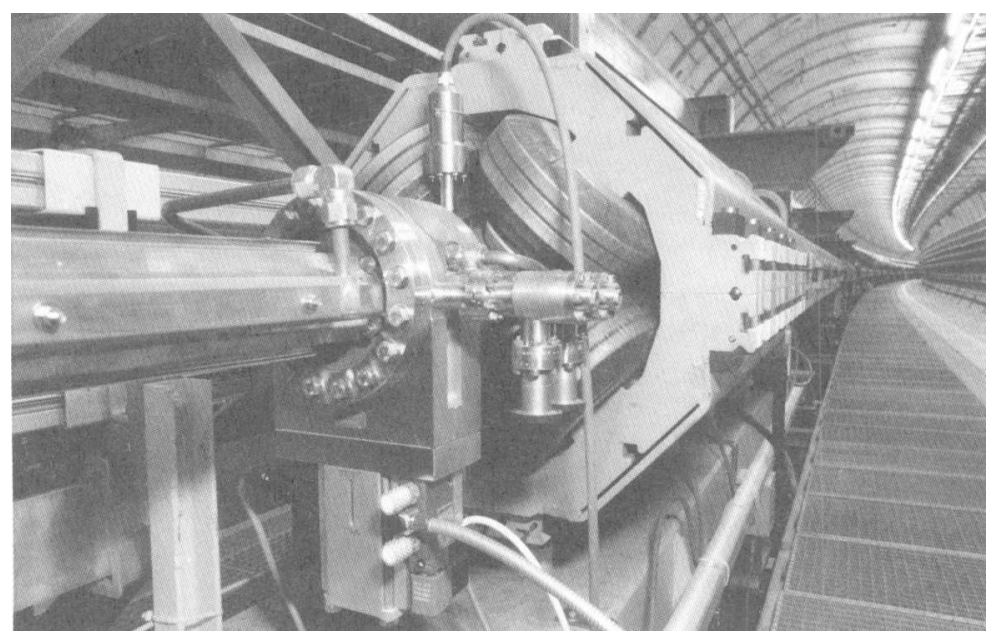

FIGURE 4: The new HERA gate valve as installed between two vacuum sections.

stability to the chamber form after the heat treatment for brazing. This low tin content does not influence significantly the other copper properties, especially its high thermal conductivity needed for dissipation of the synchrotron light power.

Besides the copper elements, the chambers are, in addition, provided with components made from stainless steel such as flanges and pump appendices, monitors, etc., which must be also brazed vacuum tightly onto the beam pipe. The total length of all vacuum tightly brazed joints for the HERA electron vacuum system amounts to about $12.5 \mathrm{~km}$. In order to increase their reliability, several techniques 
have been either newly developed, or modified. ${ }^{9}$ Among them, the most significant are the chemical cleaning of the copper elements, based on $\mathrm{H}_{2} \mathrm{O}_{2}$ instead of $\mathrm{HNO}_{3}$, and the use of brazing steps at different temperatures. ${ }^{9}$ Thus, joints between stainless steel parts are made using copper wires as braze at $1100{ }^{\circ} \mathrm{C}$ in a vacuum furnace; between copper and stainless steel with B-CuSn12 at $1000{ }^{\circ} \mathrm{C}$, also in a vacuum furnace. The final brazing between copper components is made in a special $15 \mathrm{~m}$ long furnace(Fig. 5 ) in a reducing gas atmosphere at 800 ${ }^{0} \mathrm{C}$, by using the common eutectic silver-copper braze. ${ }^{9}$ In total, 1,400 vacuum chambers, having lengths between .14 and $12 \mathrm{~m}$ were produced in a time of less than one year.

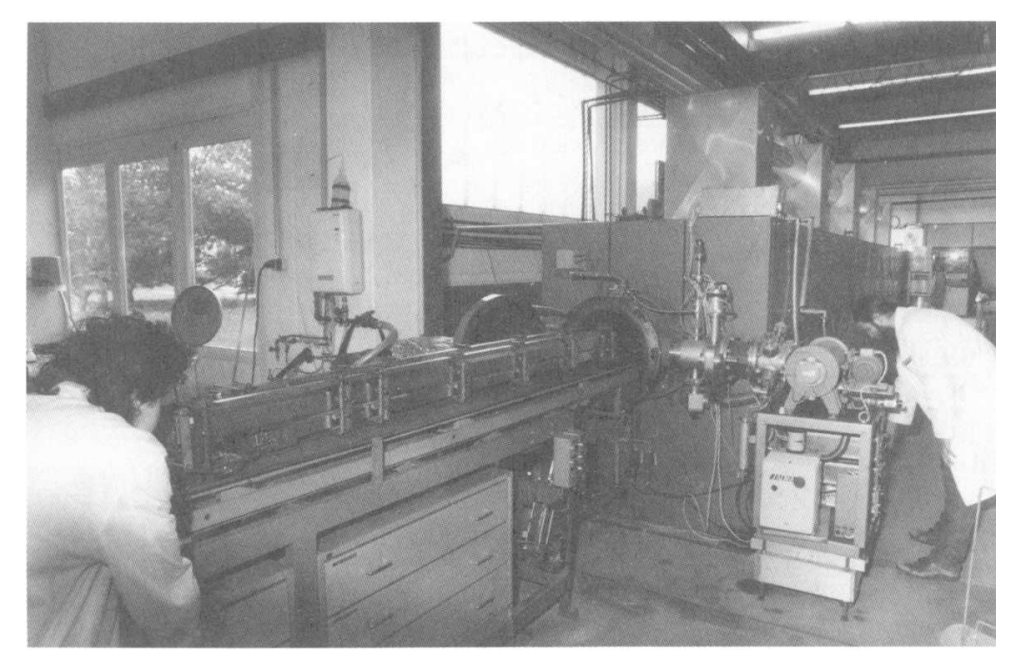

FIGURE 5 : The $15 \mathrm{~m}$ long brazing furnace used for the HERA vacuum chamber fabrication.

\section{PERFORMANCE OF THE HERA VACUUM SYSTEM}

The assembly of the HERA electron vacuum system was finished on August 9 th 1988. Ten days later, just before injecting the first electrons in HERA, the mean vacuum pressure of the systems was $5.10^{-9}$ mbar without any previous bake-out of the chambers in situ. During the following two months of tests, HERA was operated for machine studies only up to energies of $10 \mathrm{GeV}$ with beam currents usually below .3 mA. Therefore it was not possible to study the vacuum system performance at the 
maximum pumping speed of the integrated pumps, which exists at higher energies. Also the cleaning effect of the synchrotron radiation could not be studied, since the time integral of the stored current was too low.

At the end of the HERA test runs, two months later, the vacuum pressure was reduced to values lying below $10^{-9}$ mbar. The pressure increase, due to stored beam, was $9 \times 10^{-9} \mathrm{mbar} / \mathrm{mA}$ at $7 \mathrm{GeV}$. or $1.3 \times 10^{-8}$ $\mathrm{mbar} / \mathrm{mA}$ at $10 \mathrm{GeV}$. During these tests, the beam life time due to residual gas was always better than one hour.

\section{REFERENCES}

1. J. Kouptsidis, Proc. 7th Int. Vac. Congrress, pp 341-345, Vienna (1977).

2. T.W. Martin, J. Vac. Sci. Tecnol., 16(2), pp 761-762(1979).

3. R. Kohaupt, Proc. XI Intern. Conf. on High Energy Accelerators, pp 562-565, Geneva(1980).

4. R. Klatt, T. Weiland, EPAC, Europ. Particle Accelerators Conf., pp 1193-1195, Rom(1988).

5. HERA-Proposal for a large Electron-Proton Colliding Beam Facility at DESY, HERA 81/10, Hamburg(1981).

6. LEP, Design Study of a 22 to $130 \mathrm{GeV} \mathrm{e}^{+} \mathrm{e}^{-}$Colliding Beam Machine, CERN/ISR/79-35, Geneva(1979).

7. H. Hartwig, J. Kouptsidis, J. Vac. Sci. Technol., 11(6) pp 1154$1159(1974)$.

8. Barber et al, Proc. of XIII Conf. on High Energy Accelarators, Vol. 2, pp 72-75, Novosibirsk(1986).

9. Ballion et al, "Industrielle Anwendung des Hart- und Hochtemperaturloetens zur Fertigung des $6,4 \mathrm{~km}$ langen Vakuumsystems fuer den HERA-Elektronen-Speicherring ", to be published in 2nd Intern. Conf. on Brazing, High Temperature Brazing and Diffusion Welding, Essen(1989). 